\title{
INVARIANT SUBSPACES FOR DERIVATIONS
}

\author{
E. V. KISSIN
}

(Communicated by Paul S. Muhly)

\begin{abstract}
In this article it is proved that most of the known sufficient conditions for a subspace from Lat $\mathcal{A}$ to be hyperinvariant are in fact also sufficient for this subspace to be invariant for all operators from Ad $A$.
\end{abstract}

Let $A$ be an algebra of operators on a Hilbert space $H$ and let $A^{\prime}$ be its commutant. We write Lat $A$ for the lattice of closed invariant subspaces of $A$, and Hyp $A$ for the subset of all hyperinvariant subspaces in Lat $A$; i.e., all subspaces in Lat $A$ invariant for all operators from $A^{\prime}$. For the weakly closed algebra $A=A(A)$ generated by a bounded operator $A$ and the identity, Rosenthal [7] showed that if $M \in$ Lat $A$ is comparable to every point of Lat $A$, then $M \in$ Hyp $A$. A related result due to Stampfli [8] says that if $S$ is a countable subset of Lat $A$ and if every element of $S$ is comparable to every element of Lat $A \backslash S$, then $S \subseteq$ Hyp $A$. Fillmore [3] extended this result and proved that if $M$ and $N$ from Lat $A$ are comparable to every point of Lat $\mathcal{A}$ and the set $\{M, N\}=\{L \in$ Lat $A: M \subseteq L \subseteq N\}$ is countable, then $\{M, N\} \subseteq \operatorname{Hyp} A$.

In [1] Douglas and Pearcy initiated the study of topological properties of Lat $A(A)$ considered as a metric space under the metric $d(M, N)=\left\|P_{M}-P_{N}\right\|$ where $P_{M}$ and $P_{N}$ denote the orthoprojections on $M$ and $N$. They proved that if $M$ is an inaccessible point of Lat $A$, then $M \in$ Hyp $A$. In [2] they and Salinas showed that if $S$ is a totally disconnected, open subset of Lat $A$, then every subspace in $S$ is hyperinvariant. From this the results of Rosenthal, Fillmore, and Stampfli follow immediately. In [2] a result of another kind was obtained which shows the existence of a hyperinvariant subspace if Lat $A(A)$ contains two proper subspaces $M \subset N$ such that every subspace in Lat $A(A)$ is comparable with either $M$ or $N$. Murphy [6] proved that if $M, N \in \operatorname{Hyp} A$ and if $L \in\{M, N\}$ lies in no disc in $\{M, N\}$, then $L \in$ Hyp $A$.

In [4] for operator algebras $A$, the Lie algebras $\operatorname{Ad} A=\{B \in B(H):[B, A]=$ $B A-A B \in A$ for all $A \in A\}$ were considered and the triplets ( $A$, Lat $A$, Ad $A$ ) were investigated. Operators from Ad $A$ generate derivations on $A$. It is easy to see that $A$ and $A^{\prime}$ are Lie ideals in Ad $A$. Although in many important cases (such as $W^{*}$ and CSL-algebras) Ad $A=A+A^{\prime}$, very often $\operatorname{Ad} A$ is much larger than $A+A^{\prime}$ (see $[\mathbf{4}, \mathbf{5}])$.

Received by the editors April 14, 1986 and, in revised form, September 17, 1986.

1980 Mathematics Subject Classification (1985 Revision). Primary 46L15, 47A15; Secondary 47B15.

Key words and phrases. Reflexive operator algebras, topology of lattices, hyperinvariant subspaces, Lie algebra of derivations. 
By $\mathcal{L}(A)$ we denote the set of all subspaces in Lat $A$ invariant for all operators from Ad $A$. Obviously $\mathcal{L}(A) \subseteq$ Hyp $A$ and every subspace in $\mathcal{L}(A)$ is invariant for all operators in the algebra generated by $\operatorname{Ad} A$.

In this article we investigate the sufficient conditions considered in $[\mathbf{2}, \mathbf{3}, \mathbf{6}, \mathbf{7}$, 8] for a subspace or a set of subspaces from Lat $A$ to belong to Hyp $A$. We prove that these conditions are sufficient for them to belong to $\mathcal{L}(A)$. In Theorems 9 and 10 we also obtain some additional sufficient conditions for a set of subspaces from Lat $A$ to belong to $\mathcal{L}(A)$. Theorem 11 shows that if $S$ is an isolated subset of Lat $A$, then the set $S$ is invariant for Ad $A$.

Finally, I would like to thank the referee for the many helpful suggestions which have helped me to improve the article.

1. Preliminary results. Let $B(H)$ be the algebra of all bounded operators on a Hilbert space $H$. If $X$ is a set of closed subspaces of $H$, then by $\operatorname{Alg} X$ we denote the algebra of all operators in $B(H)$ which leave every member of $X$ invariant. For a bounded operator $A$ and for a subspace $M, A M$ denotes the linear subspace generated by $\{A x: x \in M\}$ and $M_{A}$ denotes $\exp (A) M$.

LEMMA 1 [1]. If $M, N$ are subspaces in $H$ and $A, B \in B(H)$ are invertible, then $d(A M, B N) \leq\|A-B\|\left(\left\|A^{-1}\right\|+\left\|B^{-1}\right\|\right)+d(M, N)\left(\left\|A^{-1}\right\|\|B\|+\left\|B^{-1}\right\|\|A\|\right)$.

Let $\mathbf{D}_{r}$ denote the disc of radius $r: \mathbf{D}_{r}=\{z \in \mathbb{C}:|z| \leq r\}$. A disc of radius $r$ in a topological space $X$ is a subset of $X$ homeomorphic to $\mathbf{D}_{r}$. Also define $\mathbf{D}_{r}(M, A)$ to be $\left\{M_{t A}:|t| \leq r\right\}$. Let $\sigma(A)$ denote the spectrum of the operator $A$.

LEMMA 2. Let $A \in B(H)$, let $M$ be a closed subspace of $H$ and let $r<$ $\ln 2 / 2\|A\|$. Then $M_{t A}$ is a closed subspace for any $t \in \mathbb{C}$ and either $A M \subseteq M$ or $\mathbf{D}_{r}(M, A)$ is homeomorphic to $\mathbf{D}_{r}$.

Proof. $M_{t A}$ is closed because $\exp (t A)$ is invertible for any $t \in \mathbb{C}$. By Lemma 1, for $t, z \in \mathbb{C}$

$$
d\left(M_{t A}, M_{z A}\right) \leq\|\exp (t A)-\exp (z A)\|(\|\exp (-t A)\|+\|\exp (-z A)\|) .
$$

Therefore the mapping $t \rightarrow M_{t A}$ is continuous.

Suppose that $M_{t A}=M_{z A}$ for some $t, z \in \mathbf{D}_{r}$. Put $u=t-z$. Then $|u| \leq 2 r$ and $M_{u A}=M$. Set $B=\exp (u A) ;$ then $B M=M$. Since $\|u A\|<\ln 2$,

$$
\|B-I\|=\left\|\sum_{n=1}^{\infty} \frac{(u A)^{n}}{n !}\right\| \leq \sum_{n=1}^{\infty} \frac{\|u A\|^{n}}{n !}=e^{\|u A\|}-1<1,
$$

and $\sigma(B)=\exp (\sigma(u A))$. Therefore $|\lambda-1|<1$ for any $\lambda \in \sigma(B)$ and

$$
u A=\ln B=\sum_{n=1}^{\infty}(-1)^{n-1}(B-I)^{n} / n .
$$

It follows from (1) that $A M \subseteq M$. Thus if $M$ is not invariant for $A$, then $t \rightarrow M_{t A}$ is a continuous and one-to-one mapping of $\mathbf{D}_{r}$ onto $\mathbf{D}_{r}(M, A)$. Since $\mathbf{D}_{r}$ is a compact subset of $\mathbb{C}, \mathbf{D}_{r}$ is homeomorphic to $\mathbf{D}_{r}(M, A)$ and the lemma is proved.

LEMMA 3 [4]. Let $A, B \in B(H)$.

(i) If $A$ is invertible, then $A \exp (B) A^{-1}=\exp \left(A B A^{-1}\right)$.

(ii) Let $\operatorname{ad} B(A)=[B, A]$. Then $\exp (B) A \exp (-B)=\exp (\operatorname{ad} B)(A)$. 
LEMMA 4. (i) Let $A$ be an operator algebra and let $B \in \operatorname{Ad} A$. The transformation $M \in$ Lat $A \rightarrow M_{B}$ is a homeomorphism of Lat $A$ onto itself.

(ii) Let $X$ be a set of closed subspaces of $H$ and let $B \in B(H)$. If $M_{t B} \in X$ for every $M \in X$ and for every $t \in \mathbb{C}$, then $B \in \operatorname{Ad}(\operatorname{Alg} X)$.

ProOF. Let $x \in M$ and $A \in \mathcal{A}$. By Lemma 3,

$$
\begin{aligned}
y & =\exp (-B) A \exp (B) x=\exp (-\operatorname{ad} B)(A) x \\
& =\sum_{k=0}^{\infty}(-1)^{k}(\operatorname{ad} B)^{k}(A) x / k ! .
\end{aligned}
$$

Since $B \in \operatorname{Ad} A$, for every $k$ the operator $(\operatorname{ad} B)^{k}(A)$ belongs to $A$ and therefore every $(\operatorname{ad} B)^{k}(A) x$ belongs to $M$. Since $M$ is closed, $y$ belongs to $M$. Therefore

$$
A \exp (B) x=\exp (B) y \in M_{B}
$$

so that $M_{B} \in$ Lat $A$. Since $\exp (B)$ is invertible, $M \rightarrow M_{B}$ is a one-to-one mapping of Lat $A$ onto itself. It follows from Lemma 1 that the mapping is continuous and the inverse mapping is also continuous, so that the mapping is a homeomorphism and (i) is proved.

For every $A \in \operatorname{Alg} X$, for every $M \in X$, and for every $t \in \mathbb{C}$,

$$
\exp (-t B) A \exp (t B) M=\exp (-t B) A M_{t B} \subseteq \exp (-t B) M_{t B}=M,
$$

since $M_{t B} \in X$. Therefore $\exp (-t B) A \exp (t B) \in \operatorname{Alg} X$. Alg $X$ is closed in the norm topology. Hence

$$
\left.\frac{d}{d t}[\exp (-t B) A \exp (t B)]\right|_{t=0}=-[B, A] \in \operatorname{Alg} X,
$$

so that $B \in \operatorname{Ad}(\operatorname{Alg} X)$, which completes the proof of the lemma.

2. Invariant and discrete subsets in Lat $A$. We say that a subset $X$ of Lat $A$ is invariant for $\operatorname{Ad} A$ if for every $M \in X$ and for every $B \in \operatorname{Ad} A$, we have that $M_{B} \in X$.

From Lemma 4 we immediately obtain the following theorem.

THEOREM 5 [4]. If $X$ is a subset of Lat $A$ invariant for Ad $A$, then $\operatorname{Ad} A \subseteq$ $\operatorname{Ad}(\operatorname{Alg} X)$. In particular $\operatorname{Ad} A \subseteq \operatorname{Ad}(\operatorname{Alg}$ Lat $A)$.

THEOREM 6. Let $M, N \in \mathcal{L}(A)$ and let $M \subset N$. If $L \in\{M, N\}$ lies in no disc in $\{M, N\}$, then $L \in \mathcal{L}(A)$.

ProOF. For any $L \in\{M, N\}$ and for $B \in \operatorname{Ad} A, L_{B} \in$ Lat $A$ and

$$
M=M_{B} \subseteq L_{B} \subseteq N_{B}=N .
$$

Therefore $\{M, N\}$ is invariant for $\operatorname{Ad} A$, so that $\mathbf{D}_{r}(L, B) \subseteq\{M, N\}$ for any positive $r$. If $L$ lies in no disc in $\{M, N\}$, then it follows from Lemma 2 that $B L \subseteq L$ for any $B \in \operatorname{Ad} A$ and the theorem is proved.

We say that a point $L \in\{M, N\}$ is inaccessible in $\{M, N\}$ if its path-component in $\{M, N\}$ is just $\{L\}$. 
COROLlaRY 7. Let $M$ and $N$ belong to $\mathcal{L}(A)$ and let $M \subset N$. If $L$ is isolated or inaccessible in $\{M, N\}$, then $L \in \mathcal{L}(A)$. In particular, if $L$ is isolated or inaccessible in Lat $\mathcal{A}$, then $L \in \mathcal{L}(\mathcal{A})$.

REMARK. In [1] it was proved that $L \in$ Hyp $\mathcal{A}$. It follows from Corollary 7 that in fact $L$ belongs to $\mathcal{L}(\mathcal{A})$.

THEOREM 8. Suppose that $S$ is a discrete subset of Lat $A$ such that the orthoprojection on every subspace in $S$ commutes with the orthoprojection on each subspace in the complement of $S$. Then every subspace in $S$ belongs to $\mathcal{L}(\mathcal{A})$.

Proof. If $M$ and $N$ are two subspaces of $H$ such that $P_{M}$ commutes with $P_{N}$, then $d(M, N)=1$. Thus $d(M, N)=1$ for every $M$ in $S$ and every $N$ in the complement of $S$. Since $S$ is discrete, every subspace in $S$ is isolated and the desired conclusion follows from Corollary 7.

REMARK. In $[\mathbf{2}, \mathbf{3}, \mathbf{7}, \mathbf{8}]$ it was proved that $S \subseteq$ Hyp $A$. From Theorem 8 it follows that in fact $S \subseteq \mathcal{L}(\mathcal{A})$.

The condition on $S$ can be made even weaker than in Theorem 8. Let $P_{M}$ be the orthoprojection on a subspace $M$ and let $Q_{M}$ be the projection on $M$ along a subspace $L$. it is easy to see that

$$
Q_{M} P_{M}=P_{M} \quad \text { and } \quad P_{M} Q_{M}=Q_{M}
$$

THEOREM 9. Suppose that $S$ is a discrete subset in Lat $A$. If for every $M$ in $S$ there exists a projection $Q_{M}$ such that every $N \in$ Lat $A \backslash S$ is invariant for $Q_{M}$, then $S \subseteq \mathcal{L}(A)$.

ProOF. Let $Q_{M}$ be the projection on $M \in S$ which satisfies the condition of the theorem and let $B \in \operatorname{Ad} A$. By Lemma 1

$$
\begin{aligned}
d\left(M_{t B}, M\right) & \leq\|\exp (t B)-I\|(\|\exp (-t B)\|+1) \\
& \leq\left(e^{\|t B\|}-1\right)\left(e^{\|t B\|}+1\right) .
\end{aligned}
$$

Since $S$ is discrete, there exists a positive $r$ such that $M_{t B} \notin S \backslash M$ for all $|t| \leq r$. By Lemma 4(i), $M_{t B} \in$ Lat $A$ and therefore $M_{t B} \in($ Lat $A \backslash S) \cup M$. Hence $Q_{M} M_{t B} \subseteq$ $M_{t B}$. From this it follows that for every $x \in M$

$$
x(t)=\exp (-t B) Q_{M} \exp (t B) x \in M .
$$

Differentiating at $t=0$ and taking into account that $M$ is closed, we obtain

$$
\left.\frac{d x(t)}{d t}\right|_{t=0}=\left[Q_{M}, B\right] x \in M,
$$

so that $M$ is invariant for $\left[Q_{M}, B\right]$. Let $P_{M}$ be the orthoprojection on $M$. Then $P_{M}\left[Q_{M}, B\right] P_{M}=\left[Q_{M}, B\right] P_{M}$. Using (2) we obtain

$$
\begin{gathered}
P_{M}\left[Q_{M}, B\right] P_{M}=P_{M} Q_{M} B P_{M}-P_{M} B Q_{M} P_{M}=Q_{M} B P_{M}-P_{M} B P_{M} \\
{\left[Q_{M}, B\right] P_{M}=Q_{M} B P_{M}-B Q_{M} P_{M}=Q_{M} B P_{M}-B P_{M} .}
\end{gathered}
$$

Therefore $P_{M} B P_{M}=B P_{M}$, so that $M \in \mathcal{L}(A)$. The theorem is proved.

Combining Theorems 5 and 9 we obtain the following general theorem. 
THEOREM 10. Let $X$ be a subset in Lat $A$ invariant for Ad $A$ and let $S$ be a discrete subset in $X$ such that for every $M \in S$ there exists a projection $Q_{M}$ on $M$ such that every $N \in X \backslash S$ is invariant for $Q_{M}$. Then $S \subseteq \mathcal{L}(\mathcal{A})$.

Proof. Let $B=\operatorname{Alg} X$. Then Lat $B=X$ and, by Theorem 9 , every $M$ in $S$ is invariant for $\operatorname{Ad} B$. By Theorem $5, \operatorname{Ad} A \subseteq \operatorname{Ad} B$, which completes the proof.

Now we shall consider a sufficient metric condition for a subset of Lat $A$ to be invariant for Ad $A$.

THEOREM 11. Let $S$ be a subset of Lat $A$ and let $m=\inf \{d(M, N): M \in$ $S, N \in$ Lat $A \backslash S\}$. If $m \neq 0$, then $S$ is invariant for Ad $A$.

Proof. Let $B \in \operatorname{Ad} A$. By (3),

$$
d\left(M_{t B}, M\right) \leq\left(e^{2\|t B\|}-1\right)
$$

and we can choose a positive $r$ such that $d\left(M_{t B}, M\right) \leq m / 2$ for all $|t| \leq r$ and for all $M \in S$. From this it follows that $M_{t B} \in S$. For any complex $\lambda$, choosing an integer $n$ so that $|\lambda / n| \leq r$ we obtain

$$
M_{\lambda B}=\exp (\lambda B) M=\exp (\lambda B / n) \cdots \exp (\lambda B / n) M \in S .
$$

Since $\lambda$ and $B$ are arbitrary, this completes the proof.

Let $\Omega$ be an open subset of Lat $A$ and let $\Gamma$ be an arcwise connected component of Lat $A$. Set

$$
\tau=\bigcap_{M \in \Omega \cap \Gamma} M \quad \text { and } \quad K=\bigvee_{M \in \Omega \cap \Gamma} M
$$

In [2, Theorem 2.2] it was proved that $\tau$ and $K$ belonged to Hyp $A$. Repeating, essentially the same argument as in [2], we obtain the following theorem.

THEOREM 12. $T$ and $\mathcal{K}$ belong to $\mathcal{L}(\mathcal{A})$.

PROOF. In [2] it was shown that it is sufficient to prove that

$$
B T \subseteq M \quad \text { and } \quad B M \subseteq \mathcal{K}
$$

for every $M \in \Omega \cap \Gamma$ and for every $B \in \operatorname{Ad} A$. Since $\Omega$ is open, repeating the same argument as in Theorem 11, we obtain that there exists a positive $r$ such that $M_{t B} \in \Omega \cap \Gamma$ for all $|t|<r$. Let $x \in \tau$. Then $x \in \bigcap_{|t|<r} M_{t B}$. Therefore for $|\lambda|<r$

$$
x(\lambda)=\exp (\lambda B) x \in \bigcap_{|t|<r} M_{(t+\lambda) B} \subseteq M .
$$

Differentiating $x(\lambda)$ at $\lambda=0$ and taking into account that $M$ is closed, we obtain that $B x \in M$. Therefore $B T \subseteq M$.

If $x \in M$, then $x(t)=\exp (t B) x \in M_{t B} \subseteq \mathcal{K}$ for all $|t|<r$. Since $\mathcal{K}$ is closed, differentiating $x(t)$ at $t=0$, we obtain that $B x \in \mathcal{K}$ which completes the proof.

Repeating the argument of $[2$, Theorem 3.1] and using Theorem 12 we immediately obtain the following theorem.

THEOREM 13. Let $A$ be an operator algebra, and suppose that there exist two nonzero proper subspaces $M$ and $N$ in Lat $A$ such that $M \subset N$ and such that 
every subspace in Lat $\mathcal{A}$ is comparable with either $M$ or $N$. Then $\mathcal{L}(\mathcal{A})$ contains a nontrivial subspace.

THEOREM 14. Let $Q_{M} \in \operatorname{Ad} A$ be the projection on a subspace $M$ along a subspace $L$. If there exists a subspace $N$ in Lat $A$ complementary to $M$, then $L$ belongs to Lat $\mathcal{A}$. If, in addition, $N$ belongs to $\mathcal{L}(\mathcal{A})$, then $L \in \mathcal{L}(\mathcal{A})$.

ProOF. If the orthoprojection $P_{M}$ on a subspace $M$ belongs to $A^{\prime}$, then $M$ and $M^{\perp}$ belong to Lat $A$. If the projection $Q_{M}$ on $M$ along a subspace $L$ belongs to $A^{\prime}$, then it is obvious that $M$ and $L$ belong to Lat $A$. Suppose now that the projection $Q_{M} \in \operatorname{Ad} A$. It is easy to see that there exists a bounded operator $T$ from $M^{\perp}$ into $M$ such that $Q_{M}=\left(\begin{array}{ll}I & T \\ 0 & 0\end{array}\right)$. Let $A=\left(A_{i j}\right) \in A$ for $1 \leq i, j \leq 2$. Then $\left[Q_{M}, A\right]$ and $\left[Q_{M},\left[Q_{M}, A\right]\right]$ belong to $A$ and it is easy to check that

$$
A_{1}=\left[Q_{M}, A\right]+\left[Q_{M},\left[Q_{M}, A\right]\right]=\left(\begin{array}{cc}
0 & B_{12} \\
0 & 0
\end{array}\right) \in \mathcal{A}
$$

where $B_{12}=2\left(A_{12}+T A_{22}-A_{11} T-T A_{21} T\right)$.

Assume now that there exists a subspace $N \in$ Lat $\mathcal{A}$ complementary to $M$, that is, $M+N=H$. It is easy to show that there exists a bounded operator $S$ from $M^{\perp}$ into $M$ such that $N=\left\{\left(\begin{array}{c}S y \\ y\end{array}\right): y \in M^{\perp}\right\}$. Then $A_{1} N \subseteq N$, so that $\left(\begin{array}{c}B_{12} y \\ 0\end{array}\right) \in N$ for all $y \in M^{\perp}$. Therefore

$$
B_{12}=2\left(A_{12}+T A_{22}-A_{11} T-T A_{21} T\right)=0 .
$$

The projection $I-Q_{M}=\left(\begin{array}{cc}0 & -T \\ 0 & I\end{array}\right)$ belongs to Ad $A$ and is the projection on the subspace $L=\left\{\left(\begin{array}{c}-T y \\ y\end{array}\right): y \in M^{\perp}\right\}$ along $M$. For every $A \in A$ and for every $z=\left(\begin{array}{c}-T y \\ y\end{array}\right) \in L$, we have that $A z=\left(\begin{array}{c}x \\ y_{1}\end{array}\right)$, where $y_{1}=\left(A_{22}-A_{21} T\right) y$ and $x=$ $\left(A_{12}-A_{11} T\right) y$. It follows from (4) that $x=-T y_{1}$, so that $L \in$ Lat $A$. If $N \in$ $\mathcal{L}(A)$, then considering $A$ from $\operatorname{Ad} A$ and using the same argument, we obtain that $L \in \mathcal{L}(A)$ which completes the proof.

COROLlaRY 15. Let an orthoprojection $P_{M}$ on $M$ belong to Ad $A$. If there exists a reducing subspace $N$ in Lat $A$ such that $M$ and $N$ are complementary $\left(d\left(M, N^{\perp}\right)<1\right)$, then $M$ is a reducing subspace in Lat $A$.

PROOF. By Theorem 14, $M^{\perp} \in$ Lat $A$. Since $N$ is a reducing subspace in Lat $A, N$ and $N^{\perp}$ belong to Lat $A$. In [1] it was proved that $M$ and $N$ are complementary if

$$
d\left(M, N^{\perp}\right)=\left\|P_{M}+P_{N}-I\right\|<1 .
$$

We have that $P_{M^{\perp}}=I-P_{M} \in \operatorname{Ad} A$ and that

$$
d\left(M^{\perp}, N\right)=\left\|I-P_{M}-P_{N}\right\|=d\left(M, N^{\perp}\right)<1,
$$

so that $M^{\perp}$ and $N^{\perp}$ are also complementary. From Theorem 14 it follows that $M$ belongs to Lat $\mathcal{A}$, so that $M$ is a reducing subspace in Lat $\mathcal{A}$.

\section{REFERENCES}

1. R. Douglas and C. Pearcy, On a topology for invariant subspaces, J. Funct. Anal. 2 (1968), 323-341.

2. R. Douglas, C. Pearcy, and N. Salinas, Hyperinvariant subspaces via topological properties of lattices, Michigan Math. J. 20 (1973), 109-113. 
3. P. Fillmore, Notes on operator theory, Van Nostrand Reinhold, New York, 1970.

4. E. V. Kissin, On some reflexive algebras of operators and the operator Lie algebras of their derivations, Proc. London Math. Soc. (3) 49 (1984), 1-35.

5. __ On some reflexive operator algebras constructed from two sets of closed operators and from a set of reflexive algebras, Pacific J. Math. 126 (1987), 125-143.

6. G. J. Murphy, Hyperinvariant subspaces and the topology on Lat A, Pacific J. Math. 110 (1984), 183-190.

7. P. Rosenthal, A note on unicellular operators, Proc. Amer. Math. Soc. 19 (1968), 505-506.

8. J. G. Stampfli, On hyponormal and Toeplitz operators, Math. Ann. 183 (1969), 328-336.

Department of Mathematics, Statistics and Computing, The Polytechnic OF NORTH LONDON, HOLLOWAY, LONDON N7 8DB, ENGLAND 